\title{
Advection, diffusion, and delivery over a network
}

\author{
Luke L. M. Heaton, ${ }^{1,2}$ Eduardo López, ${ }^{2,3}$ Philip K. Maini, ${ }^{3,4,5}$ Mark D. Fricker, ${ }^{3,6}$ and Nick S. Jones ${ }^{2,3,5,7, *}$ \\ ${ }^{1}$ LSI DTC, Wolfson Building, University of Oxford, Parks Road, Oxford OX1 3QD, United Kingdom \\ ${ }^{2}$ Physics Department, Clarendon Laboratory, University of Oxford, Parks Road, Oxford OX1 3PU, United Kingdom \\ ${ }^{3}$ CABDyN Complexity Centre, Saïd Business School, University of Oxford, Park End Street, Oxford OXI 1HP, United Kingdom \\ ${ }^{4}$ Centre for Mathematical Biology, Mathematical Institute, University of Oxford, 24-29 St Giles', Oxford OXI 3LB, United Kingdom \\ ${ }^{5}$ Oxford Centre for Integrative Systems Biology, Department of Biochemistry, University of Oxford, Oxford OX1 3QU, United Kingdom \\ ${ }^{6}$ Department of Plant Sciences, University of Oxford, South Parks Road, Oxford OXI 3RB, United Kingdom \\ ${ }^{7}$ Department of Mathematics, Imperial College, London SW7 2AZ, United Kingdom
}

(Received 6 June 2011; revised manuscript received 16 May 2012; published 7 August 2012)

\begin{abstract}
Many biological, geophysical, and technological systems involve the transport of a resource over a network. In this paper, we present an efficient method for calculating the exact quantity of the resource in each part of an arbitrary network, where the resource is lost or delivered out of the network at a given rate, while being subject to advection and diffusion. The key conceptual step is to partition the resource into material that does or does not reach a node over a given time step. As an example application, we consider resource allocation within fungal networks, and analyze the spatial distribution of the resource that emerges as such networks grow over time. Fungal growth involves the expansion of fluid filled vessels, and such growth necessarily involves the movement of fluid. We develop a model of delivery in growing fungal networks, and find good empirical agreement between our model and experimental data gathered using radio-labeled tracers. Our results lead us to suggest that in foraging fungi, growth-induced mass flow is sufficient to account for long-distance transport, if the system is well insulated. We conclude that active transport mechanisms may only be required at the very end of the transport pathway, near the growing tips.
\end{abstract}

DOI: 10.1103/PhysRevE.86.021905

PACS number(s): 87.19.rh, 05.60.Cd, 47.63.Jd

\section{INTRODUCTION}

Many biological, geophysical, and technological systems involve the transport of material over a network by advection and diffusion [1-5]. Indeed, fluid transport systems are found in the vast majority of multicellular organisms, as the component cells of such organisms require resources for metabolism and growth, and diffusion alone is only an effective means of exchange at microscopic length scales [6]. Molecules of interest are carried by advection and diffusion through the mycelial networks of fungi $[7,8]$, the cardiovascular networks of animals [5,9-16], the xylem and phloem elements of tracheophytes (vascular plants) [17-21], and various body cavities of many different animals. Advection and diffusion are also fundamental to transport in geological and technological systems, such as rivers and drainage networks [22], gas pipelines, sewer systems, and ventilation systems $[9,23]$.

In all of these cases, the particles of interest diffuse within a moving fluid, which is constrained to flow within a given network. The bulk movement of fluid is referred to as advection, convection, or mass flow, and in general the fluid in question travels with a mean velocity that varies significantly from one part of the network to another. For example, the velocity of human blood drops from $1 \mathrm{~m} \mathrm{~s}^{-1}$ in the aorta to around $1 \mathrm{~mm} \mathrm{~s}^{-1}$ in the capillaries [24,25]. Digital imaging technologies have enabled scientists to obtain detailed knowledge of the structure of vascular networks [26], and given a network and a distribution of velocities, we may wish to calculate how an initial distribution of a resource changes over time. That is the general problem that our method is

*nick.jones@ imperial.ac.uk designed to solve, whether we are considering nutrients in a fungal network [7,8], pollutants in a drainage network [2-4], or drugs in the cardiovascular system [9-15].

The translocation of a resource within fungal networks is much less well studied than transport in the other major multicellular kingdoms of life, but the ability of fungal colonies to translocate resources is a critical feature of many ecosystems [27-29]. The relative roles of mass flow (advection), diffusion, and active transport are very poorly understood. Fungal systems have the benefit that the network is accessible, so development can be readily followed through a time series of images [30-32] and resource distribution can be mapped using scintillation imaging techniques [32-35]. In this paper, we consider the particular case of modeling the translocation of the ${ }^{14} \mathrm{C}$-labeled amino-acid analog, $\alpha$ aminoisobutyric acid (AIB), in a fungal network, where new edges in the network grow over time, edges disappear through a process of autophagy (self-eating), and edges thicken or thin over time.

Our modeling approach is to suppose that resources are consumed or delivered out of the network at a given local rate, while the resource that remains in the network moves by advection and diffusion. In the general case, we suppose that there is perfect mixing at the nodes, and each edge $i j$ in a network has a local delivery rate $R_{i j}$, a cross-sectional area $S_{i j}$, a length $l_{i j}$, a mean velocity of fluid flow $u_{i j}$, and a dispersion coefficient $D_{i j}$. The local delivery rate $R_{i j}$ represents the probability per unit time that any given unit of resource will be consumed, lost, or delivered out of the network. Koplik et al. [36] describe an effective method for calculating the exact moments of the transit times for a neutral tracer across an arbitrary network that contains a flowing medium, but which initially contains no tracer. We have advanced their methods to handle arbitrary initial conditions, noting that over any time 
step, the resource in a given edge either reaches one of the nodes at either end of the edge, or it remains within the given edge. Fick's law and the conservation of resources relates the quantity of material passing through each of the nodes, and these principles have enabled us to formulate a mixed method that couples a network-based method with a partial differential equation (PDE) for each individual edge.

The outline of this paper is as follows: Preliminary assumptions and the fundamental equations governing advection, diffusion, and delivery are discussed (Secs. II A and II B). We then outline possible methods for numerically solving such a system of equations (Sec. II C), before describing our preferred approach. By applying the Laplace transform, we identify the solution of the fundamental equation governing a single edge in the case of zero initial conditions (Sec. III A), and this method of solution is then applied to a network with multiple edges (Sec. III B). We then consider the case of nonzero initial conditions (Secs. IV A and IV B), and use that analysis to develop an algorithm for updating the exact quantity of the resource in each segment of a network as these quantities vary over time, subject to advection, diffusion, and local resource delivery (see Sec. V). A more detailed analysis of this method of solution is presented in the Supplemental Material [37], where we also describe the Gaver-Stehfest algorithm for inverting our solutions from Laplace space into the time domain.

In Sec. VI, we note that fungal growth involves changes in volume which requires the movement of fluid. For example, the cytoplasm in a growing hyphal tube moves forward with the growing tip [38], and as aqueous fluids are effectively incompressible, growth requires the net movement of fluid from the sites of water uptake to the sites of growth [30]. Moreover, given both the relative conductance and the volumetric rate of growth of different parts of a fungal network, we can calculate a unique set of currents which are consistent with the observed growth, and which also minimize the work required to overcome viscous drag. Given this set of currents, the molecular diffusion coefficient of the tracers, and the rate at which they are delivered out of the transport pathway, we can employ the algorithm described in Sec. V to calculate the expected concentration of tracers in each part of the network over time. We found good empirical agreement between our model and experimental data gathered using radio-labeled tracers, which suggests that in foraging fungi, growth-induced mass flow is sufficient to account for most long-distance transport.

\section{FURTHER DETAILS}

\section{A. Preliminary assumptions}

We are interested in calculating the distribution of a resource across a network of tubes, where the resource in question has a molecular diffusion coefficient $D_{m}$, and where we are given four essential properties for each edge in the network (see Fig. 1). The edge connecting nodes $i$ and $j$ has the following:

(1) a cross-sectional area, denoted $S_{i j}$.

(2) a length, denoted $l_{i j}$. As the location of the nodes does not vary over time, $l_{i j}$ is constant.

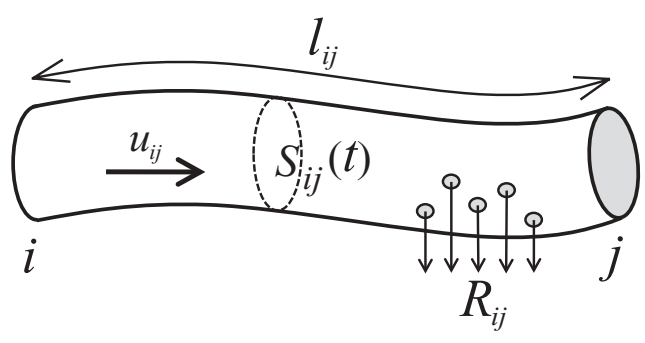

FIG. 1. Properties of a single edge in a resource distribution network. $S_{i j}$ denotes the cross-sectional area of edge $i j$ at time $t$, $l_{i j}$ denotes the length of the edge, the resource and medium flows along the edge with a mean velocity $u_{i j}$, and the resource is delivered out of the network at a rate $R_{i j}$. Note that the resource travels along each edge (and into other edges) by advection and diffusion, but the total rate at which the resource in the edge is delivered out of the network is simply $R_{i j}$ times the quantity of the resource present in the edge. Also note that we do not need to assume that the edges in our network are straight, but we do assume that a single length scale $l_{i j}$ captures the distance that the particles must travel to move from $i$ to $j$.

(3) a mean velocity, denoted $u_{i j}$. This represents the mean velocity of the fluid in the edge, and we say that $u_{i j}$ is positive if and only if the current flows from node $i$ to node $j$ (so $u_{i j}=-u_{j i}$ ).

(4) Finally, we suppose that the resource in edge $i j$ is delivered out of the network at a rate $R_{i j}$, so if a particle is in $i j$ for a short period of time $\Delta t$, then the probability that it is delivered out of the network in that time is $R_{i j} \Delta t$.

As we are interested in quantifying motion along the length of the edge $i j$, we represent each edge as a one-dimensional object, while each node is represented as a point. The term $u_{i j}$ tells us the mean speed at which particles travel from node $i$ towards node $j$, but while there is a global value for the molecular diffusion coefficient $D_{m}$, the dispersion coefficient $D_{i j}$ may be different for each edge. That is because the value of $D_{i j}$ captures the tendency of adjacent particles to spread out along the length $i j$. This number may differ from the diffusion coefficient in one dimension because particles not only diffuse along the length of the transport vessels that comprise the edge $i j$, but they also spread out because they can diffuse between the slow moving fluid by the edge of the vessels and the relatively fast moving fluid in the center of each vessel.

If we consider the case where each edge $i j$ is composed of some number of cylindrical tubes of radius $r_{i j}$ (see Fig. 2), and if the Reynold's number is small, then we can calculate $D_{i j}$ by using Taylor's dispersion coefficient for laminar flow in a cylindrical tube [39]. This formula tells us that

$$
D_{i j}=D_{m}+u_{i j}^{2} \frac{r_{i j}^{2}}{48 D_{m}}
$$

In the case of a mammalian vascular network, $r_{i j}$ is simply the lumen radius of the edge $i j$. In other networks, including fungal networks, each edge can be modeled as a bundle of cylindrical tubes, in which case $r_{i j}$ is the characteristic radius of the component transport vessels and $S_{i j}$ is the total crosssectional area of the transport vessels. 


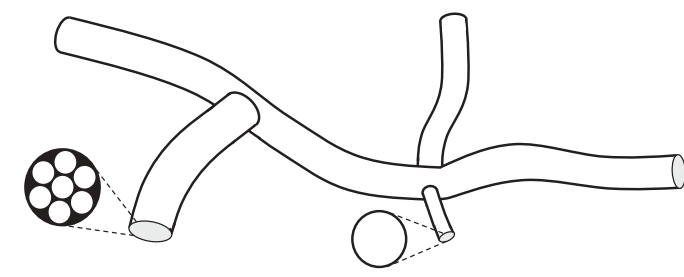

FIG. 2. Properties of an arbitrary resource distribution network. Each edge in the network is comprised of a single vessel or a bundle of transport vessels, and each edge has a length $l_{i j}$, a total cross-sectional area $S_{i j}$, a mean velocity of flow $u_{i j}$, and a local delivery rate $R_{i j}$. Each edge also has a dispersion coefficient $D_{i j}$, as described by Eq. (1). Note that the values of $D_{i j}$ depend on the molecular diffusion coefficient $D_{m}$, the velocities $u_{i j}$, and the radius of the transport vessels within the edge $i j$. The nodes represent the point of contact between the edges: we assume that there is perfect mixing at each node, and we require a consistent concentration at node $i$, whether we consider it to be one end of edge $i j$ or one end of any other edge connected to node $i$.

\section{B. Fundamental equations}

We suppose that the resource is delivered to cells while it moves across the network by advection and diffusion, resulting in changing concentrations at every point. We will only consider longitudinal coordinates along the edge $i j$, using real numbers $x$ to denote distances from node $i$, where $0 \leqslant x \leqslant l_{i j}$. Each edge contains a quantity of resource, which must satisfy the one-dimensional advection-diffusion-delivery equation

$$
\frac{\partial q_{i j}}{\partial t}+R_{i j} q_{i j}+u_{i j} \frac{\partial q_{i j}}{\partial x}-D_{i j} \frac{\partial^{2} q_{i j}}{\partial x^{2}}=0,
$$

where $q_{i j}$ is the quantity of resource per unit length, $u_{i j}$ is the mean velocity, $D_{i j}$ is the dispersion coefficient, and $R_{i j}$ is the rate at which a unit of resource is lost or delivered out of the network. In other words, at time $t$ and location $x$, the amount of the resource in a $\Delta x$ long slice of the edge is $q_{i j}(x, t) \Delta x$. The distribution of the resource within each edge will vary over space and time, but if there is no direct link between the nodes $i$ and $j$, then we let $S_{i j}=0$ and $q_{i j}(x, t)=0$ to indicate the absence of an edge. This ensures that the sums in the following equations are properly defined for all pairs of nodes $i$ and $j$.

Crucially, the concentration at node $i$ must be consistent across the edges $i j, i k$, etc., and we let $c_{i}(t)$ denote the concentration at node $i$ at time $t$ (amount of resource per unit volume). For each edge $i j$, node $i$ is located at point $x=0$ and node $j$ is located at point $x=l_{i j}$, so where $S_{i j}$ denotes the cross-sectional area of edge $i j$, we have

$$
c_{i}(t)=\frac{q_{i j}(0, t)}{S_{i j}} \text { and } c_{j}(t)=\frac{q_{i j}\left(l_{i j}, t\right)}{S_{i j}} .
$$

We also assume that there is perfect mixing at the nodes, and that the nodes have an infinitesimal volume. In other words, the edge $i j$ is only affected by the rest of the network via the concentrations at nodes $i$ and $j$.

It follows from our assumptions that the concentration profile in edge $i j$ is completely determined by Eq. (2) together with the initial condition $q_{i j}(x, 0)$ and the boundary conditions $S_{i j} c_{i}(t)$ and $S_{i j} c_{j}(t)$. By Fick's law, the rate at which a resource leaves node $i$ along edge $i j$ is given by

$$
J_{i j}(t)=\left[u_{i j} q_{i j}(x, t)-D_{i j} \frac{\partial q_{i j}(x, t)}{\partial x}\right]_{x=0} .
$$

Our framework can accommodate the case where a resource is introduced at node $i$ at some given rate $I_{i}(t)>0$. If node $i$ is not an inlet node (that is, a point where the resource enters the network), then the quantity of the resource entering node $i$ is equal to the quantity of the resource leaving node $i$, so we have $I_{i}(t)=0$. In either case, Eq. (4) implies that the net rate at which the resource leaves node $i$ is

$$
I_{i}(t)=\sum_{j}\left[u_{i j} q_{i j}(x, t)-D_{i j} \frac{\partial q_{i j}(x, t)}{\partial x}\right]_{x=0} .
$$

Note that we may be concerned with both the quantity of the resource and the quantity of fluid that passes a given point per unit of time. Henceforth the term current is reserved for the quantity of a resource that passes a given point per unit of time, while medium current refers to the volume of the advecting medium that passes a given point per unit of time: a quantity described by the term $S_{i j} u_{i j}$.

\section{Alternative methods}

Given a network and a system of equations of the form of Eq. (2), there are several methods that could be used to find the solution. We could generate an estimate of the solution by taking a particle-based approach, where a large number of particles move across the network and the path taken by each particle is determined probabilistically, as is the time taken to travel from one node to the next [4]. The problem with such particle-based approaches is the challenge of avoiding undersampling in the regions of the network that contain a low concentration of a resource. This problem occurs because, in a finite simulation, the low probability paths are of course less well sampled, but the fact that such regions are part of the network may exert a significant effect on the movement of the resource, particularly on the higher moments of the transit times for particles moving across the network $[36,40,41]$. Indeed, that is why the dispersion of tracers can be used to probe the structure of networks, and why tracer dispersion plays such a critical role in geophysical surveying techniques $[3,4]$.

Another possible approach is to employ a finite difference scheme. However, in a network where the transport velocities vary over several orders of magnitude, the straightforward applications of such an approach are not efficient. The problem is that the time scale for updating the concentrations is essentially determined by the fastest edge; for stability, the distance traveled by advection per time step must be smaller than the spatial resolution (i.e., the Courant number must be less than one). Using such a small time step may be very inefficient in the slower moving regions of the network $[42,43]$.

\section{ADVECTION, DIFFUSION, AND DELIVERY IN LAPLACE SPACE}

Given our system of fundamental equations, we want to find the quantity of the resource throughout the network, which may vary over time. It is convenient to follow the approach 
of [36], which entails the application of the Laplace transform. This operation convolves the different time scales over which the resource may move from one node to another, so it is an efficient way to handle the wide range of velocities our network may contain. In particular, we take advantage of the following properties of the Laplace transform $\mathcal{L}\left(q_{i j}(x, t)\right)=$ $\int_{0}^{\infty} q_{i j}(x, t) e^{-s t} d t=Q_{i j}(x, s)$ :

$$
\begin{gathered}
\mathcal{L}\left(\frac{\partial q_{i j}(x, t)}{\partial t}\right)=s Q_{i j}(x, s)-q_{i j}(x, 0), \\
\mathcal{L}\left(\frac{\partial q_{i j}(x, t)}{\partial x}\right)=\frac{\partial}{\partial x} Q_{i j}(x, s) .
\end{gathered}
$$

If $u_{i j}$ and $D_{i j}$ are constant over time, then it follows from Eq. (2) that

$$
\left(s+R_{i j}\right) Q_{i j}+u_{i j} \frac{\partial Q_{i j}}{\partial x}-D_{i j} \frac{\partial^{2} Q_{i j}}{\partial x^{2}}=q_{i j}(x, 0) .
$$

Furthermore, Eqs. (5)-(7) imply that

$$
\Upsilon_{i}(s)=\sum_{j}\left[u_{i j} Q_{i j}(x, s)-D_{i j} \frac{\partial}{\partial x} Q_{i j}(x, s)\right]_{x=0},
$$

where $\Upsilon_{i}(s)$ denotes the Laplace transform of $I_{i}(t)$, the net current flowing out of node $i$.

\section{A. Zero initial conditions in an edge}

We begin by considering an initially empty edge, before extending our results to the more complex case of nonzero initial conditions. We let $q_{i j}(x, 0)=0$, and consider the homogeneous case for Eq. (8):

$$
\left(s+R_{i j}\right) Q_{i j}+u_{i j} \frac{\partial Q_{i j}}{\partial x}-D_{i j} \frac{\partial^{2} Q_{i j}}{\partial x^{2}}=0 .
$$

By solving this ordinary differential equation (ODE) in the usual manner, we find that for some pair of constants $A$ and $B$,

$$
Q_{i j}(x, s)=A e^{\frac{u_{i j}+\alpha_{i j}(s)}{2 D_{i j}} x}+B e^{\frac{u_{i j}-\alpha_{i j}(s)}{2 D_{i j}} x},
$$

where

$$
\alpha_{i j}(s)=\sqrt{u_{i j}^{2}+4 D_{i j}\left(s+R_{i j}\right)} .
$$

Note that the Laplace variable $s$ represents a rate, and that $\alpha_{i j}(s)=\alpha_{j i}(s)$ is positive, and dimensionally equivalent to speed. Roughly speaking, $\alpha_{i j}(s)$ represents the speed at which a resource travels over the time scale $1 / s$, with a correction term to account for delivery.

Equation (11) tells us that for any positive number $s$, we can find $A$ and $B$ and express $Q_{i j}(x, s)$ in terms of the quantity of the resource at either end of the edge. For any given $s$, we denote the quantity of the resource at the ends of each edge by

$$
\begin{aligned}
& X_{i j}(s) \equiv Q_{i j}(0, s), \\
& X_{j i}(s) \equiv Q_{j i}(0, s)=Q_{i j}\left(l_{i j}, s\right) .
\end{aligned}
$$

For each edge $i j$, it is convenient to define two, dimensionless ratios between time scales:

$$
g_{i j}=\frac{u_{i j} l_{i j}}{2 D_{i j}} \quad \text { and } \quad h_{i j}(s)=\frac{\alpha_{i j}(s) l_{i j}}{2 D_{i j}} .
$$

Setting $x=0$ and $x=l_{i j}$ tells us that

$$
\begin{aligned}
& X_{i j}=A+B, \\
& X_{j i}=A e^{\left(g_{i j}+h_{i j}\right)}+B e^{\left(g_{i j}-h_{i j}\right)} .
\end{aligned}
$$

$A, B, X_{i j}, X_{j i}, \alpha_{i j}$, and $h_{i j}$ are all functions of the Laplace variable $s$, but this dependence is omitted for the sake of clarity, and we omit the subscript $i j$ from the terms $g_{i j}, h_{i j}$, and $l_{i j}$. Equation (15) tells us that

$$
A e^{(g+h)}+\left(X_{i j}-A\right) e^{(g-h)}=X_{j i},
$$

so

$$
A=\frac{X_{j i} e^{-g}-X_{i j} e^{-h}}{2 \sinh (h)}
$$

and likewise

$$
B=\frac{X_{i j} e^{h}-X_{j i} e^{-g}}{2 \sinh (h)} .
$$

Assuming that edge $i j$ is initially empty, we can find $Q_{i j}(x, s)$ by substituting Eqs. (14), (16), and (17) into Eq. (11), giving us

$$
\begin{aligned}
Q_{i j}(x, s)= & X_{i j} \frac{\sinh \left(\frac{l-x}{l} h\right)}{\sinh (h)} e^{\frac{x}{l} g} \\
& +X_{j i} \frac{\sinh \left(\frac{x}{l} h\right)}{\sinh (h)} e^{\frac{x-l}{l} g} .
\end{aligned}
$$

\section{B. Advection, diffusion, and delivery in an initially empty, static network}

Having examined the case of a single edge, we now turn to the problem of coupling the edges of a network such that the concentrations vary continuously as we move from one edge to another. For each node $i$, we have $C_{i}(s)=\int_{0}^{\infty} c_{i}(t) e^{-s t} d t$. Assuming that the cross-sectional areas $S_{i j}$ are constant, Eqs. (3) and (13) imply that for all edges $i j$ we have

$$
C_{i}(s)=\frac{X_{i j}(s)}{S_{i j}} \text { and } C_{j}(s)=\frac{X_{j i}(s)}{S_{i j}} .
$$

The enforcement of this equation ensures that the Laplace transform of the concentration at node $i$ is consistent for all edges $i j, i k$, and so on. In general, we may not know the Laplace transform of the node concentrations $\bar{C}(s)=\left\{C_{1}(s), \ldots, C_{m}(s)\right\}$, where $m$ is the number of nodes. However, given $\bar{I}(t)=\left\{I_{1}(t), \ldots, I_{m}(t)\right\}$ (the net current of the resource leaving each node), we can calculate $\bar{\Upsilon}(s)=$ $\left\{\Upsilon_{1}(s), \ldots, \Upsilon_{m}(s)\right\}$ (the Laplace transform of $\bar{I}$ ), and, in the following manner, calculate $\bar{C}(s)$. Substituting Eq. (11) into Eq. (9) tells us that

$$
\Upsilon_{i}(s)=\sum_{j} \frac{\alpha_{i j}(s)}{2}(B-A)+\frac{u_{i j}}{2}(A+B) .
$$

Equations (14) and (15) imply that $A+B=X_{i j}$, and

$$
\begin{aligned}
B-A= & \frac{1}{2 \sinh \left[h_{i j}(s)\right]}\left[X_{i j} e^{h_{i j}(s)}-X_{j i} e^{-g_{i j}}\right. \\
& \left.-X_{j i} e^{-g_{i j}}+X_{i j} e^{-h_{i j}(s)}\right],
\end{aligned}
$$


so we have

$$
\begin{aligned}
\Upsilon_{i}(s)= & \sum_{j}\left(\frac { \alpha _ { i j } ( s ) } { 2 \operatorname { s i n h } [ h _ { i j } ( s ) ] } \left\{X_{i j} \cosh \left[h_{i j}(s)\right]\right.\right. \\
& \left.\left.-X_{j i} e^{-g_{i j}}\right\}+\frac{u_{i j}}{2} X_{i j}\right) .
\end{aligned}
$$

Equations (19) and (20) imply that

$$
\begin{aligned}
\Upsilon_{i}(s)= & \sum_{j}\left(C_{i}(s) S_{i j}\left\{\frac{u_{i j}}{2}+\frac{\alpha_{i j}(s)}{2 \tanh \left[h_{i j}(s)\right]}\right\}\right. \\
& \left.-C_{j}(s) S_{i j}\left\{\frac{\alpha_{i j}(s) e^{-g_{i j}}}{2 \sinh \left[h_{i j}(s)\right]}\right\}\right) .
\end{aligned}
$$

In other words, for each node $i$, we have a linear equation in $C_{1}(s), C_{2}(s), \ldots, C_{m}(s)$. Hence where $\bar{C}(s)$ and $\bar{\Upsilon}(s)$ are column vectors, we have

$$
\mathbf{M}(s) \bar{C}(s)=\bar{\Upsilon}(s),
$$

where

$$
\mathbf{M}_{i j}(s)= \begin{cases}\sum_{k} S_{i k}\left\{\frac{u_{i k}}{2}+\frac{\alpha_{i k}(s)}{2 \tanh \left[h_{i k}(s)\right]}\right\} & \text { if } i=j, \\ \frac{-S_{i j} \alpha_{i j}(s) e^{-g_{i j}}}{2 \sinh \left[h_{i j}(s)\right]} & \text { otherwise. }\end{cases}
$$

We refer to the matrix $\mathbf{M}(s)$ as the propagation matrix, and it contains a row and column for each node in the given network. Given $\mathbf{M}(s)$ and $\bar{\Upsilon}(s)$, we can calculate $\bar{C}(s)$ using various efficient algorithms, including the stabilized biconjugate gradient method (BiCGStab). In most cases, this is the most efficient algorithm to use, as our matrix $\mathbf{M}(s)$ is nonsymmetric and sparse [44].

Equation (12) implies that the diagonal elements $\mathbf{M}(s)$ are all positive. Furthermore, $\mathbf{M}_{i j}(s)=0$ if and only if there is no edge between $i$ and $j$, and the other off-diagonal elements are negative. We note that if there is a resource at node $j$, then it may be transported along $i j$, bringing the resource to $i$ and reducing $\Upsilon_{i}(s)$ (the Laplace transform of the net current flowing out of node $i$ ). The resource can only reach node $i$ along the edges $i j, i k$, etc., so $\Upsilon_{i}(s)$ is completely determined by the concentration at $i$ and the concentrations that flow through the nodes adjacent to $i$. As $\Upsilon_{i}(s)$ is the Laplace transform of the net current flowing out of node $i$, and the resource at nodes $j \neq i$ can flow into node $i$, the off-diagonal elements of $\mathbf{M}(s)$ are negative, and zero if $i$ and $j$ are not directly connected.

Multiplying $\left|\mathbf{M}_{i j}(s)\right|$ by $C_{j}(s)$ gives us the Laplace transform of the current of the resource flowing from $j$ to $i$, so, roughly speaking, $\left|\mathbf{M}_{i j}(s)\right|$ represents the size of the volumetric current from $j$ to $i$, over the time scale $1 / s$. Note that if $u_{i j}$ is positive, then the medium current flows from $i$ to $j$, $\left|\mathbf{M}_{i j}(s)\right|<\left|\mathbf{M}_{j i}(s)\right|$, and there is a greater flow from $i$ to $j$ than the other way around. That is to say, when the medium current is from $i$ to $j$, the value of $C_{i}(s)$ has a greater influence on the value of $\Upsilon_{j}(s)$ than the influence of $C_{j}(s)$ on the value of $\Upsilon_{i}(s)$. Also note that the ratio of $\mathbf{M}_{i j}(s)$ to $\mathbf{M}_{j i}(s)$ depends on the Péclet number of the edge, $\frac{u_{i j} l_{i j}}{D_{i j}}=2 g_{i j}[9,36]$, as $\mathbf{M}_{i j}(s): \mathbf{M}_{j i}(s)$ is equal to $1: e^{2 g_{i j}}$.

For very short time scales, we have a very large $s$, and by Eq. (12), $\alpha_{i j} \gg u_{i j}$ and $\alpha_{i j} \approx \sqrt{4 D_{i j} s}$. In this case, the off-diagonal elements of $\mathbf{M}$ are very small, and $\mathbf{M}_{i i} \approx$ $\sum_{k} S_{i k} \frac{\alpha_{i k}}{2} \approx \sum_{k} S_{i k} \sqrt{D_{i k} s}$. In other words, over very short time scales, the resource is lost from the nodes by a process of diffusion, but it does not have time to reach the other nodes. Over longer time scales, the difference between $u_{i j}$ and $\alpha_{i j}$ is smaller, the off-diagonal elements of $\mathbf{M}$ are larger, and the effect of advection is greater.

\section{NONZERO INITIAL CONDITIONS}

\section{A. Nonzero initial conditions in a single edge}

We now consider advection, diffusion, and delivery along a single edge $i j$, where the initial condition $q_{i j}(x, 0)$ is nonzero. We let the length of $i j$ equal $l$, the longitudinal dispersion coefficient is $D$, the local delivery rate is $R$, and the mean velocity is $u$.

We have seen that for any positive Laplace constant $s$, $Q_{1}(x, s)=e^{(g+h) \frac{x}{l}}$ and $Q_{2}(x, s)=e^{(g-h) \frac{x}{l}}$ satisfy the homogeneous analog, given by Eq. (10). Furthermore, the Wronskian

$$
\begin{aligned}
W_{i j}(x, s) & =Q_{1}(x, s) \frac{\partial Q_{2}(x, s)}{\partial x}-\frac{\partial Q_{1}(x, s)}{\partial x} Q_{2}(x, s) \\
& =\frac{-\alpha e^{2 g \frac{x}{l}}}{D} .
\end{aligned}
$$

By the method of variation of parameters, for any given $s$ and any given initial condition $q_{i j}(y, 0)$, the following function $f\left(x, s, q_{i j}(y, 0)\right)$ is a particular solution to the fundamental Eq. (8):

$$
\begin{aligned}
f\left(x, s, q_{i j}(y, 0)\right)= & \frac{e^{(g-h) \frac{x}{l}}}{\alpha} \int_{0}^{x} e^{(h-g) \frac{y}{l}} q_{i j}(y, 0) d y \\
& -\frac{e^{(g+h) \frac{x}{l}}}{\alpha} \int_{0}^{x} e^{-(g+h) \frac{y}{l}} q_{i j}(y, 0) d y .
\end{aligned}
$$

Note that $f(0, s, q)=0$ for all initial conditions $q$. Also note that if $q=q_{1}+q_{2}$, then $f(x, s, q)=f\left(x, s, q_{1}\right)+f\left(x, s, q_{2}\right)$. Since $f\left(x, s, q_{i j}(y, 0)\right)$ is a particular solution of Eq. (8), for each edge $i j$, there is a pair of constants $A$ and $B$ such that

$$
Q_{i j}(x, s)=A e^{(g+h) \frac{x}{l}}+B e^{(g-h) \frac{x}{l}}+f\left(x, s, q_{i j}(y, 0)\right) .
$$

Because $f(0, s, q)=0$ for all initial conditions $q$, Eqs. (14) and (25) imply that

$$
\begin{gathered}
X_{i j} \equiv Q_{i j}(0, s)=A+B \\
X_{j i} \equiv Q_{i j}\left(l_{i j}, s\right)=A e^{(g+h)}+B e^{(g-h)}+f\left(l, s, q_{i j}(y, 0)\right) .
\end{gathered}
$$

We can therefore express $A$ and $B$ in terms of $X_{i j}$ and $X_{j i}$. Indeed, substituting Eq. (26) into Eq. (27) and multiplying both sides by $e^{-g}$ tells us that

$$
X_{j i} e^{-g}=A\left(e^{h}-e^{-h}\right)+X_{i j} e^{-h}+e^{-g} f\left(l, s, q_{i j}(y, 0)\right) .
$$

For any edge $i j$, we let

$$
\beta_{i j}(s) \equiv \frac{-\alpha_{i j}(s) e^{-g_{i j}}}{2 \sinh \left[h_{i j}(s)\right]} f\left(l, s, q_{i j}(y, 0)\right),
$$

and explain its physical significance in Sec. IV B. 
In the case of our single edge, Eqs. (26) and (28) imply that

$$
\begin{aligned}
& A=\frac{X_{j i} e^{-g}-X_{i j} e^{-h}}{2 \sinh (h)}+\frac{\beta_{i j}(s)}{\alpha}, \\
& B=\frac{X_{i j} e^{h}-X_{j i} e^{-g}}{2 \sinh (h)}-\frac{\beta_{i j}(s)}{\alpha} .
\end{aligned}
$$

The substitution of these results into Eq. (25) tells us that for any initial condition $q_{i j}(y, 0)$,

$$
\begin{aligned}
Q_{i j}(x, s)= & {\left[\frac{X_{j i} e^{-g}-X_{i j} e^{-h}}{2 \sinh (h)}+\frac{\beta_{i j}(s)}{\alpha}\right] e^{(g+h) \frac{x}{l}} } \\
& +\left[\frac{X_{i j} e^{h}-X_{j i} e^{-g}}{2 \sinh (h)}-\frac{\beta_{i j}(s)}{\alpha}\right] e^{(g-h) \frac{x}{l}} \\
& +f\left(x, s, q_{i j}(y, 0)\right) .
\end{aligned}
$$

\section{B. Nonzero initial conditions over a network}

Having analyzed the case of a single edge with a nonzero initial condition, we now consider an entire network and find an exact solution that ensures that for all $t>0$, the concentration varies continuously as we move from one edge to another. The first step in finding this solution is to note that Eq. (24) implies that

$$
\begin{aligned}
\frac{\partial f\left(x, s, q_{i j}\right)}{\partial x}= & -\frac{(u+\alpha)}{2 D \alpha} e^{(g+h) \frac{x}{l}} \int_{0}^{x} e^{-(g+h) \frac{y}{l}} q_{i j}(y, 0) d y \\
& +\frac{(u-\alpha)}{2 D \alpha} e^{(g-h) \frac{x}{l}} \int_{0}^{x} e^{(h-g) \frac{y}{l}} q_{i j}(y, 0) d y,
\end{aligned}
$$

where for the sake of clarity we drop the subscript $i j$ from $u_{i j}$, $\alpha_{i j}, l_{i j}, g_{i j}, h_{i j}$, and $D_{i j}$, and ignore the dependence on $s$ of the terms $\alpha_{i j}$ and $h_{i j}$. Note that for any initial condition $q_{i j}(y, 0)$, we have $\left.\frac{\partial f\left(x, s, q_{i j}(y, 0)\right)}{\partial x}\right|_{x=0}=0$. It follows that

$$
\begin{aligned}
\left.\frac{\partial Q_{i j}(x, s)}{\partial x}\right|_{x=0}= & \frac{\beta_{i j}(s)}{D}+\frac{u+\alpha}{2 D}\left[\frac{X_{j i} e^{-g}-X_{i j} e^{-h}}{2 \sinh (h)}\right] \\
& +\frac{u-\alpha}{2 D}\left[\frac{X_{i j} e^{h}-X_{j i} e^{-g}}{2 \sinh (h)}\right] .
\end{aligned}
$$

Now, recall that $\Upsilon_{i}(s)$ denotes the Laplace transform of the net current of resource flowing away from node $i$, and that $\Upsilon_{i}(s)=0$ unless $i$ is an inlet node. Substituting Eq. (32) into Eq. (9) gives us

$$
\begin{aligned}
\Upsilon_{i}(s)= & \sum_{j} X_{i j}\left\{\frac{u_{i j}}{2}+\frac{\alpha_{i j}(s)}{2 \tanh \left[h_{i j}(s)\right]}\right\} \\
& -\sum_{j} X_{j i} \frac{\alpha_{i j}(s) e^{-g_{i j}}}{2 \sinh \left[h_{i j}(s)\right]}-\sum_{j} \beta_{i j}(s) .
\end{aligned}
$$

As we assume that the cross-sectional areas $S_{i j}$ are constant, Eqs. (19) and (33) imply that

$$
\begin{aligned}
\Upsilon_{i}(s)= & C_{i}(s) \sum_{j} S_{i j}\left\{\frac{u_{i j}}{2}+\frac{\alpha_{i j}(s)}{2 \tanh \left[h_{i j}(s)\right]}\right\} \\
& -\sum_{j} C_{j}(s) S_{i j} \frac{\alpha_{i j}(s) e^{-g_{i j}}}{2 \sinh \left[h_{i j}(s)\right]}-\sum_{j} \beta_{i j}(s) .
\end{aligned}
$$

In matrix form, we have

$$
\mathbf{M}(s) \bar{C}(s)=\bar{p}(s),
$$

where

$$
\begin{gathered}
\bar{C}(s)=\left\{C_{1}(s), C_{2}(s), \ldots, C_{m}(s)\right\}^{\mathrm{T}}, \\
p_{i}(s)=\Upsilon_{i}(s)+\sum_{j} \beta_{i j}(s),
\end{gathered}
$$

and $\mathbf{M}(s)$ is the propagation matrix as in Eq. (23). Note that the effect of the initial conditions on the concentration at the nodes is completely captured by the terms $\beta_{i j}(s)$, and that, as before, the propagation matrix $\mathbf{M}(s)$ relates the concentrations at the nodes to the net currents flowing out of the nodes. Furthermore, by comparison with Eq. (23), we can see that the concentration at the nodes is the same as would be the case if the network were initially empty, and the Laplace transform of the net current leaving node $i$ were $p_{i}(s)$ rather than $\Upsilon_{i}(s)$.

In effect, the formalism of the propagation matrix enables us to substitute an initial condition in the edges around node $i$ for a boundary condition at node $i$. For each node $i$ and each Laplace variable $s$, this boundary condition is of the form $\sum_{j} \beta_{i j}(s)$. Intuitively speaking, the term $\beta_{i j}(s)$ represents the Laplace transform of the quantity of the resource that first leaves edge $i j$ by arriving at node $i$. Note that we have not calculated the impact of the initial condition $q_{i j}(x, 0)$ on the future concentration profile $q_{i j}(x, t)$ for $t>0$ : we have simply calculated the impact of the initial conditions on the concentrations at the nodes (see Sec. V).

\section{EFFICIENT CALCULATION OF RESOURCE DISTRIBUTION}

If we wish to find the concentration at various points in the network other than the nodes, there are two ways we can proceed. The first method is to suppose that each edge contains several nodes in addition to its terminal points. The problem with this approach is that introducing additional nodes increases the size of the propagation matrix, and finding $\bar{C}(s)$ by inverting the matrices $\mathbf{M}(s)$ is the major computational cost of the propagation matrix algorithm. Furthermore, although this approach can be used to find the concentration at each of a given set of points, it does not provide a means of finding the quantity of a resource between a given pair of points. We could approximate the total quantity of a resource between two points by assuming that the concentration varies in a linear manner from one point to the next, but as the exact solution may contain boundary layer effects, we might require a very high spatial resolution to ensure that such a linear approximation is accurate.

A different approach, which we take, calculates the total quantity of the resource within each section of the network, regardless of the spatial resolution. The key conceptual step involves partitioning the resource into two parts. We can imagine that the resource is composed of a large number of particles, and over a given time scale, each particle in edge $i j$ either reaches node $i$ or $j$, or it does not. We let $\hat{q}_{i j}(x, t)$ denote the quantity of the resource per unit length at the point 
$0 \leqslant x \leqslant l_{i j}$ in edge $i j$ and time $t$, where a given particle only contributes to $\hat{q}_{i j}(x, t)$ if it has passed through a node (any node) by time $t$ after initialization. More precisely, we work in Laplace space and let $\mathcal{L}\left(\hat{q}_{i j}(x, t)\right)=\hat{Q}_{i j}(x, s)$. This term denotes the Laplace transformed concentration profile that would occur if the network was initially empty, and if the Laplace transform of the net current leaving each node was $p_{i}(s)=\Upsilon_{i}(s)+\sum_{j} \beta_{i j}(s)$, rather than $\Upsilon_{i}(s)$.

As we have seen, the impact of the initial condition on the concentration at the nodes is completely captured by the terms $\beta_{i j}(s)$. However, $\hat{Q}_{i j}(x, s)$ and $\hat{q}_{i j}(x, t)$ do not fully capture the influence of the initial condition $q_{i j}(x, 0)$ on the concentration profile $q_{i j}(x, t)$ for $t>0$. In addition to $\hat{q}_{i j}(x, t)$ (the quantity of resource that has reached a node over the time scale $t$ ), we must also consider the resource that starts in edge $i j$, and which does not reach node $i$ or $j$ over the time scale $t$. We let $\tilde{q}_{i j}(x, t)$ denote the quantity of such a resource at the point $0 \leqslant x \leqslant l_{i j}$ in edge $i j$ and time $t$, where by definition

$$
\tilde{q}_{i j}(x, t)=q_{i j}(x, t)-\hat{q}_{i j}(x, t) .
$$

We can calculate the concentration at each node by calculating $\beta_{i j}(s)$ for every $i$ and $j$, and by using the propagation matrix. Furthermore, because at time 0 none of the resource in edge $i j$ has had time to reach a node, we can apply Eq. (18), and find $\hat{Q}_{i j}(x, s)$ in terms of the boundary conditions $X_{i j}(s)$ and $X_{j i}(s)$. Given $\hat{Q}_{i j}(x, s)$ for $s=\frac{\ln 2}{t}, \ldots, n \frac{\ln 2}{t}$, we can apply the Gaver-Stehfest algorithm [37], which inverts our solution from Laplace space into the time domain. In addition to finding $\hat{q}_{i j}(x, t)$ (the quantity of the resource at each point that has passed through any of the nodes), we also solve a separate PDE for each edge, which tells us how the resource that stays within each edge has evolved over a given time step $t$. That is to say, for each edge $i j$, we find $\tilde{q}_{i j}(x, t)$, given that $\tilde{q}_{i j}(x, t)$ satisfies the fundamental advection-diffusion-delivery Eq. (2), $\tilde{q}_{i j}(x, 0)=q_{i j}(x, 0), \tilde{q}_{i j}(0, t)=0$, and $\tilde{q}_{i j}\left(l_{i j}, t\right)=0$. Finally, Eq. (37) tells us that $q_{i j}(x, t)=\tilde{q}_{i j}(x, t)+\hat{q}_{i j}(x, t)$.

In particular, we consider the case where the initial condition is stepwise constant, and edge $i j$ is divided into $N_{i j}$ sections of equal length. That is to say, we suppose that for all $1 \leqslant n \leqslant N$, we have

$$
q_{i j}(x, 0)=k_{i j}^{(n)} \text { for all } x_{n-1}<x<x_{n} .
$$

As we wish to update these quantities over time, we let $k_{i j}^{(n)}(t)$ denote the mean quantity of a resource per unit length in the $n$th section at the given time $t$. For any $t>0$, we can find an exact solution for the updated mean quantities per unit length,

$$
k_{i j}^{(n)}(t)=\frac{N_{i j}}{l_{i j}} \int_{\frac{n-1}{N_{i j}} l_{i j}}^{\frac{n}{N_{i j}} l_{i j}} q_{i j}(x, t) d x .
$$

Finally, we note that this algorithm can be adapted for the case where the cross-sectional areas and other parameters vary over time, though our method requires that the lengths $l_{i j}$, mean velocities $u_{i j}$, decay rates $R_{i j}$, and dispersion coefficients $D_{i j}$ are either constant or vary in a stepwise manner. In the case where we wish to find the concentration of resource in a growing fungal network (see Sec. VI), we simply vary all of the parameters in a stepwise manner, finding the distribution of the resource at the end of each time step, and treating that distribution as an initial condition for the following time step.

\section{CONCENTRATION IN A GROWING FUNGAL NETWORK}

Multicellular organisms need to supply individual cells with the resources necessary for survival, but while transport in animals and plants is relatively well studied, surprisingly little is known about transport in the third major kingdom of multicellular life. The fungal body or mycelium can be understood as a network of fluid filled tubes or hyphae, which grow by osmotically drawing water from their surroundings while adding material to the cell wall specifically at the tips of the growing hyphae [45-48]. Diffusion may be sufficient to sustain short-range local growth when resources are abundant, but foraging fungi such as Phanerochaete velutina can grow hundreds of millimeters away from a food source over metabolically inert surfaces [7,49,50]. Together with other experimental evidence, this observation strongly suggests that long-distance transport mechanisms are required to deliver nutrients to the growing tips at a sufficient rate, though there are many open questions concerning the mechanism(s) of transport $[7,8,30,50,55,56]$. Vesicles moved by motor proteins, contractile elements, diffusion, and carefully regulated osmotic gradients have all been proposed as mechanisms for driving long-range transport in fungi [7,8,50-54]. Though a fundamental physiological question, the issue of which (if any) of these mechanisms is important continues to remain a subject for debate. Our model suggests that the minimum currents consistent with the observed growth can account for much of the observed movement of the radio tracers. We also note that the movement of fluid towards the growing margin does not require the coordination of concentration gradients across the mycelium.

\section{A. Measuring networks and predicting currents}

To obtain a sequence of digitized fungal networks, we placed a wood block inoculated with $P$. velutina in a microcosm of compacted sand [see Fig. 3(a)]. The growing mycelium was photographed every three days, and the sequence of images was manually marked to record the location of nodes or junctions, as well as the presence or absence of edges in the fungal network. These edges were not sufficiently well resolved to make direct measurements of their diameter from the digitized images. However, the reflected intensity, averaged over a small user-defined kernel at either end of the edge, correlated well with microscope-based measurements of edge thickness. The observed relationship between image intensity and thickness was therefore used to estimate edge thickness across the whole network [49].

After the final photograph of the network was taken, we assessed the pattern of nutrient transport by adding ${ }^{14} \mathrm{C}$-AIB to the inoculum. A semitranslucent scintillation screen was gently placed onto the fungal network and imaged using a photon-counting camera [32-35]. As AIB is not metabolized, the ${ }^{14} \mathrm{C}$ label faithfully reports the distribution of the aminoacid analog, and so the density of photons arriving from each region of the network can be used to estimate the 
distribution of AIB [see Fig. 3(b)]. To obtain intensity values for each edge, the digitized network was aligned with the image from the photon-counting camera. The empirically determined intensities could then be compared with the predictions of an advection-diffusion-delivery (ADD) model, which we describe in this section.

The edges in the fungal networks are composed of bundles of hyphae and transport vessels bounded by an outer rind [57]. Unlike individual hyphae, the cords (or edges) in a fungal network have hydrophobic coatings, which insulate them from the environment $[7,8]$. Since the mycelium is composed of incompressible material, the rate of increase in the volume of each edge must equal the volumetric rate of flow into that edge minus the volumetric rate of flow out of that edge. In order to model the movement of radio-labeled AIB in a growing fungal network, we make the following assumptions:

(1) We assume that all of the water and other materials which form the mycelium ultimately originate from the inoculum [see Fig. 3(a)].

(2) Mass flows are assumed to occur in transport vessels of radius $6 \mu \mathrm{m}$, which occupy some fraction $\lambda$ of the crosssectional area of each edge. Note that this assumption implies that the hydraulic conductance of each edge is proportional to its cross-sectional area, as the number of transport vessels in each edge is proportional to its cross-sectional area.

(3) Growing edges are sinks for fluid, while shrinking edges and the inoculum are sources. It is assumed that each edge continues to grow or shrink at the rate that was measured over the final time step. Given the distribution of sources and sinks, and given the relative hydraulic conductance of each edge, we can use basic circuit theory to calculate the currents across the mycelium.

(4) We let the molecular diffusion coefficient $D_{m}=3.5 \times$ $10^{-6} \mathrm{~cm}^{2} \mathrm{~s}^{-1}$, which is the diffusion coefficient of AIB in water [9], and we use Eq. (1) to calculate the dispersion coefficient $D_{i j}$ for each edge.

(5) We assume that radio-labeled AIB enters the network at the inoculum at a constant rate, and as AIB is not metabolized (and there is negligible decay of ${ }^{14} \mathrm{C}$ ), we let the local delivery rate $R=0.05$ day $^{-1}$, though very similar results are obtained for any small value of $R$. The rate of AIB uptake at the inoculum does not affect the relative concentration of points in the network, and we assume that the number of photons leaving node $i$ between times $T$ and $T+\tau$ is proportional to $\int_{T}^{T+\tau} c_{i}(t) d t$, where $c_{i}(t)$ denotes the concentration at node $i$ at time $t$.

We let $c_{i}^{\prime}(t)=c_{i}(T+t)$, and note that by definition, $\int_{T}^{T+\tau} c_{i}(t) d t$ is equivalent to the convolution of $c_{i}^{\prime}(t)$ with the unit function. The convolution theorem [58] therefore implies that

$$
\mathcal{L}\left(\int_{0}^{\tau} c_{i}(t) d t\right)=\frac{1}{s} \mathcal{L}\left(c_{i}^{\prime}(\tau)\right) .
$$

The method described in Sec. V enables us to find $C_{i}(s)=$ $\mathcal{L}\left(c_{i}^{\prime}(\tau)\right)$ for $s=\frac{\ln 2}{\tau}, \ldots, n \frac{\ln 2}{\tau}$, and so, by Eq. (40), we can find $\mathcal{L}\left(\int_{T}^{T+\tau} c_{i}(t) d t\right)$ for $s=\frac{\ln 2}{\tau}, \ldots, n \frac{\ln 2}{\tau}$. We can therefore use the Gaver-Stehfest algorithm to calculate $\int_{T}^{T+\tau} c_{i}(t) d t$, and we assume that the mean concentration in edge $i j$ is halfway between the mean concentrations at nodes $i$ and $j$.
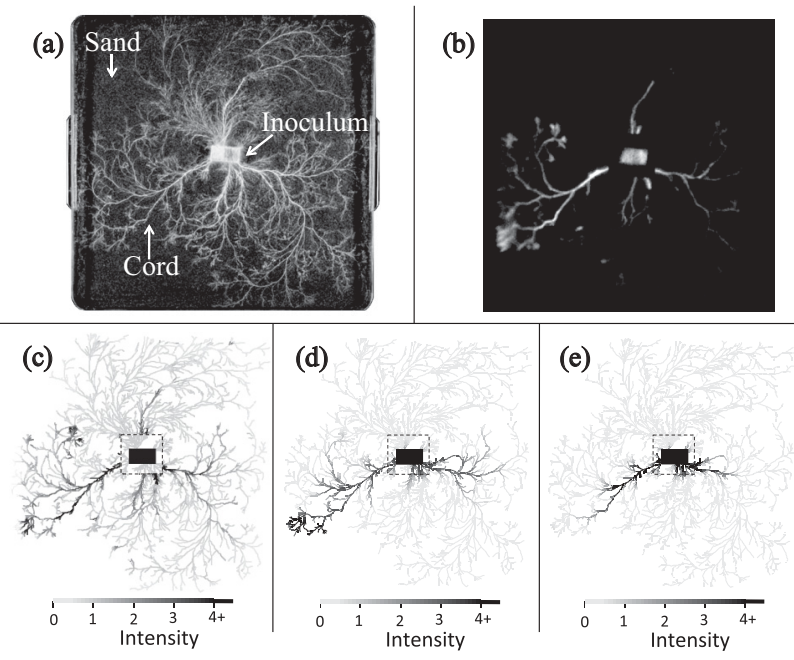

FIG. 3. Measured and predicted intensities. (a) Microcosm and mycelium of Phanerochaete velutina, photographed just before radiolabeled AIB was dripped onto the inoculum. (b) Data from the photoncounting camera. The brightness of the image reflects the total number of photons emitted from each region over the period of time starting 5.6 hours after the AIB was added, and finishing 37.6 hours after the AIB was added. (c) Digitized network, colored to indicate the density of photons reaching the camera from each region of the network. Luminosity is measured in arbitrary units, and the edges inside the dotted line were not covered by the scintillation screen. (d) Predicted intensity measured in arbitrary units, under the assumptions that AIB enters the network at the inoculum at a constant rate, each edge in the final network continues to grow (or shrink) at the same rate that was observed over the final time step, and $\lambda=0.1$ (so we assume that $10 \%$ of each edge is occupied by transport vessels). (e) Predicted intensity in arbitrary units under the same assumptions as diagram (d), except that in this case we let $\lambda=0.2$.

\section{B. Results}

The mycelium formed a branching network spanning most of the microcosm. The distribution pattern for ${ }^{14} \mathrm{C}$-AIB was remarkably asymmetric, with high levels in part of the network growing in the lower left quadrant, lower levels in the lower right quadrant, and almost none in the upper quadrants, apart from one short section of cord. The explanation for this behavior is not readily apparent from the static architecture of the network, as regions with a low label still have substantial cords. However, when the growth of the network over the last time step is considered using the ADD model, there is an impressive degree of congruency between the model predictions for different values of $\lambda$ and the empirical determination of nutrient distribution (see Fig. 3).

We find that $29 \%$ of points emit photons at a rate greater than the mean background intensity plus three standard deviations. In the case of the ADD model, where we set $\lambda=0.1,27 \%$ of the points in the network are predicted to have a mean intensity that is significantly greater than zero. That fraction drops to $17 \%$ when we let $\lambda=0.2$. In a duplicate experiment, $28 \%$ of points contain enough AIB to produce a significant signal, compared to a prediction of $32 \%$ when $\lambda=0.1$ and $18 \%$ when $\lambda=0.2$. By setting $\lambda$ such that our model predicts the correct number of points with a significant amount of AIB, we find that $\lambda \approx 0.1$ in both replicates. Note that we can vary 
TABLE I. Pearson's linear correlation coefficient $\rho$ between the predicted intensity and the empirical data. By way of comparison, we can calculate the Euclidean distance from each point to the center of the inoculum, and the value of $\rho$ between those distances and the empirical data was -0.27 in Experiment 1 and -0.28 in Experiment 2.

\begin{tabular}{lcc}
\hline \hline$\lambda$ & Experiment 1 & Experiment 2 \\
\hline 0.05 & 0.45 & 0.38 \\
0.10 & 0.56 & 0.31 \\
0.15 & 0.56 & 0.31 \\
0.20 & 0.55 & 0.30 \\
\hline \hline
\end{tabular}

the number of points that are predicted to contain a significant amount of the resource by varying the free parameter $\lambda$, but for any reasonable value of $\lambda$, our model is successful at predicting which points will contain a significant amount of resource (see Fig. 3). To quantify the accuracy of our model predictions, we can calculate the Pearson's linear correlation coefficient $\rho$ between the measured intensity of each edge and the predicted intensity. For comparison, we can also calculate the value of $\rho$ between the measured intensity of each edge and the distance from that edge to the inoculum. We found that our model's predictions are more accurate than simply assuming that the concentration at each point is a linear function of the distance from the source. Furthermore, the best fit between our model's prediction and the empirical data arises when we let $\lambda \approx 0.1$ (see Table I).

\section{CONCLUSION}

In this paper, we have presented a method for calculating the concentration of a resource that arises when a given material is subject to advection, diffusion, and local delivery out of the transport network (see Sec. V). Nature is full of networks in which materials within a fluid are transported by advection and diffusion while being consumed or delivered, so these algorithms have many potential applications. As a scientifically significant example, we employed our algorithm to implement a model of transport in a growing fungal network (see Sec. VI). The expansion of fluid filled vessels requires the movement of fluid, and we have developed a model for calculating the scale of growth-induced mass flows in each edge of a given network. We found good empirical agreement between experimental data and the concentrations predicted by our model, and this agreement was best when we set the free parameter $\lambda \approx 0.1$.

The claim that $10 \%$ of each cord is occupied by transport vessels is biologically plausible [57], and we suspect that agreement between the ADD model and the empirical data was better in the first experiment because in the second experiment the mycelium had reached the edges of the microcosm by the time the AIB was added, which made it more difficult to accurately measure growth. We are not claiming that $10 \%$ of each cord is occupied by transport vessels, or that growthinduced mass flows are the only transport process occurring within fungi. Our method of imaging only captures cords, and not the growth of fine hyphae, so we expect that our model underestimates the scale of growth and growth-induced mass flows [30]. Furthermore, a more detailed analysis indicates that active transport mechanisms may be needed near the growing margin, where the mean velocity of the cytosol is similar to the velocity of tip growth [59]. Nevertheless, our results suggest that when water uptake and growth are spatially separated, the mass flows that inevitably occur can make a dominating contribution to long-range fungal transport (see Sec. VI).

\section{ACKNOWLEDGMENTS}

L.L.M.H. thanks the EPSRC for financial support and thanks Sid Redner for his helpful comments. E.L. received financial support via EPSRC Grant No. EP/E056997/1. P.K.M. was partially supported by a Royal Society Wolfson Research Merit Award, and Award No. U54CA143970 from the National Cancer Institute. M.D.F. thanks the BBSRC and NERC. N.S.J. received financial support via EPSRC Grants No. EP/I005765/1 and No. EP/I005986/1.
[1] A. Bunde and S. Havlin, Fractals and Disordered Systems (Springer-Verlag, Berlin, 1991).

[2] D. Ben-Avraham and S. Havlin, J. Phys. A 15, 691 (1982).

[3] H. A. Makse, J. S. Andrade, and H. E. Stanley, Phys. Rev. E 61, 583 (2000).

[4] M. Sahimi, H. T. Davis, and L. E. Scriven, Chem. Eng. Commun. 23, 329 (1983).

[5] J. P. Kirkpatrick, D. M. Brizel, and M. W. Dewhirst, Radiat. Res. 159, 336 (2003).

[6] M. LaBarbera, Science 249, 992 (1990).

[7] J. W. G. Cairney, Mycol. Res. 96, 135 (1992).

[8] D. H. Jennings, Biol. Rev. 62, 215 (1987).

[9] G. A. Truskey, F. Yuan, and D. F. Katz, Transport Phenomena in Biological Systems (Pearson, Upper Saddle River, New Jersey, 2010).

[10] D. Goldman and A. S. Popel, J. Theor. Biol. 206, 181 (2000).

[11] D. Goldman, Microcirculation 15, 795 (2008).
[12] R. J. Shipley and S. J. Chapman, Bull. Math. Biol. 72, 1464 (2010).

[13] T. F. Sherman, J. Gen. Physiol. 78, 431 (1981).

[14] D. A. Beard and J. B. Bassingthwaighte, Ann. Biomed. Eng. 28, 253 (2000).

[15] D. A. Beard and J. B. Bassingthwaighte, Ann. Biomed. Eng. 29, 298 (2001).

[16] D. Szczerba and G. Székely, J. Theor. Biol. 234, 87 (2005).

[17] L. Sack and N. M. Holbrook, Ann. Rev. Plant Biol. 57, 361 (2006).

[18] M. V. Thompson, Trends Plant Sci. 11, 1360 (2005).

[19] K. A. McCulloh, J. S. Sperry, and F. R. Adler, Nature (London) 421, 939 (2003).

[20] P. S. Nobel, Physicochemical and Environmental Plant Physiology (Academic, San Diego, 1991).

[21] J. H. Young, R. F. Evert, and W. Eschrich, Planta 113, 355 (1973) 
[22] J. Banavar, A. Maritan, and A. Rinaldo, Nature (London) 408, 159 (2000).

[23] G. West, J. Brown, and B. Enquist, Science 276, 122 (1997).

[24] P. J. Daley, K. B. Sagar, and L. S. Wann, Brit. Heart J. 54, 562 (1985).

[25] P. Butti, M. Intaglietta, H. Reimann, C. Holliger, A. Bollinger, and M. Anliker, Microvasc. Res. 10, 220 (1975).

[26] E. Bullitt, K. Muller, I. Jung, W. Lin, and S. Aylward, Med. Image Anal. 9, 39 (2005).

[27] J. Deacon, Fungal Biology, 2nd ed. (Blackwell, Oxford, 2005).

[28] G. M. Gadd, ed., Fungi in Biogeochemical Cycles (Cambridge University Press, Cambridge, UK, 2006).

[29] O. B. Lindahl and A. F. S. Taylor, Plant Soil 242, 123 (2002).

[30] L. L. M. Heaton, E. López, P. K. Maini, M. D. Fricker, and N. S. Jones, Proc. R. Soc. B 277, 3265 (2010).

[31] L. L. M. Heaton, B. Obara, V. Grau, N. S. Jones, T. Nakagaki, L. Boddy, and M. D. Fricker, Fungal Biol. Rev. 26, 12 (2012).

[32] M. D. Fricker, J. Lee, D. P. Bebber, M. Tlalka, and J. Hynes, J. Microsc. 231, 317 (2008).

[33] M. Tlalka, S. C. Watkinson, P. R. Darrah, and M. D. Fricker, New Phytol. 153, 173 (2002).

[34] M. Tlalka, D. Hensman, P. R. Darrah, S. C. Watkinson, and M. D. Fricker, New Phytol. 158, 325 (2003).

[35] S. C. Watkinson, L. Boddy, K. Burton, P. R. Darrah, D. Eastwood, M. D. Fricker, and M. Tlalka, Mycologist 19, 11 (2005)

[36] J. Koplik, S. Redner, and D. Wilkinson, Phys. Rev. A 37, 2619 (1988).

[37] See Supplemental Material at http://link.aps.org/supplemental/ 10.1103/PhysRevE.86.021905 where we detail the mathematical machinery involved in solving the advection-diffusiondelivery equation over a network, including the Gaver-Stehfest algorithm.

[38] R. R. Lew, Microbiology 151, 2685 (2005).
[39] G. Taylor, Proc. R. Soc. A 219, 186 (1953).

[40] S. Redner, A Guide to First-Passage Processes (Cambridge University Press, Cambridge, UK, 2001).

[41] L. de Arcangelis, J. Koplik, S. Redner, and D. Wilkinson, Phys. Rev. Lett. 57, 996 (1986).

[42] R. Courant, K. Friedrichs, and H. Lewy, Math. Ann. 100, 215 (1928).

[43] G. D. Smith, Numerical Solution of Partial Differential Equations: Finite Difference Methods (Oxford University Press, Oxford, UK, 1985).

[44] H. A. V. der Vorst, SIAM J. Sci. Stat. Comput. 13, 631 (1992).

[45] S. Bartnicki-Garcia, C. E. Bracker, G. Gierz, R. López-Franco, and H. Lu, Biophys. J. 79, 2382 (2008).

[46] N. Money, Fungal Biol. Rev. 22, 71 (2008).

[47] G. Steinberg, Eukaryotic Cell 6, 351 (2006).

[48] S. Bartnicki-Garcia, D. D. Bartnicki, and G. Gierz, Can. J. Bot. 73, 372 (1995).

[49] D. P. Bebber, J. Hynes, P. R. Darrah, L. Boddy, and M. D. Fricker, Proc. R. Soc. B 274, 2307 (2007).

[50] S. Olsson and S. N. Gray, FEMS Microbiol. Ecol. 26, 109 (1998).

[51] A. Yamamoto, J. Cell Sci. 116, 4501 (2003).

[52] R. Suelmann, N. Sievers, and R. Fischer, Mol. Microbiol. 25, 757 (1997).

[53] G. Steinberg, Fungal Genet. Biol. 24, 161 (1998).

[54] P. Darrah, M. Tlalka, A. Ashford, and S. Watkinson, Eukaryotic Cell 5, 1111 (2006).

[55] G. P. Boswell, H. Jacobs, F. Davidson, G. Gadd, K. Ritz, and F. A. Davidson, Mycologist 17, 165 (2003).

[56] R. R. Lew, Nature Rev. Mol. 9, 509 (2011).

[57] D. Eamus, W. Thompson, J. Cairney, and D. Jennings, J. Exp. Bot. 36, 1110 (1985).

[58] E. Kreyszig, Advanced Engineering Mathematics, 9th ed. (Wiley, New York, 2006).

[59] L. L. M. Heaton, Ph.D. thesis, University of Oxford, 2012. 